4 Grootendorst DC, Gauw SA, Benschop N, et al. Efficacy of the novel phosphodiesterase-4 inhibitor BAY 19-8004 on lung function and airway inflammation in asthma and chronic obstructive pulmonary disease (COPD). Pulm Pharmacol Ther 2003; 16: 341-347.

5 Hatzelmann A, Morcillo EJ, Lungarella G, et al. The preclinical pharmacology of roflumilast - a selective, oral phosphodiesterase 4 inhibitor in development for chronic obstructive pulmonary disease. Pulm Pharmacol Ther 2010; 23: 235-256.

6 Grootendorst DC, Gauw SA, Verhoosel RM, et al. Reduction in sputum neutrophil and eosinophil numbers by the PDE4 inhibitor roflumilast in patients with COPD. Thorax 2007; 62: 1081-1087.

7 Gamble E, Grootendorst DC, Brightling CE, et al. Antiinflammatory effects of the phosphodiesterase-4 inhibitor cilomilast (Ariflo) in chronic obstructive pulmonary disease. Am J Respir Crit Care Med 2003; 168: 976-982.

8 Homma S, Sakamoto T, Hegab AE, et al. Association of phosphodiesterase $4 \mathrm{D}$ gene polymorphisms with chronic obstructive pulmonary disease: relationship to interleukin 13 gene polymorphism. Int J Mol Med 2006; 18: 933-939.

9 Barber R, Baillie GS, Bergmann R, et al. Differential expression of PDE4 cAMP phosphodiesterase isoforms in inflammatory cells of smokers with COPD, smokers without COPD, and nonsmokers. Am J Physiol Lung Cell Mol Physiol 2004; 287: L332-L343.

\title{
Why excessive sleepiness may persist in OSA patients receiving adequate CPAP treatment
}

\section{To the Editors:}

We read with interest the paper by VERNET et al. [1], which describes the characteristics of residual excessive sleepiness (RES) in obstructive sleep apnoea (OSA) patients treated with continuous positive airway pressure (CPAP). The authors studied two groups of OSA patients, with and without RES. The apnoea/hypopnoea index dramatically decreased in both groups, testifying that CPAP was applied properly. Patients underwent cognitive, psychological, biological and clinical tests, and 24-h sleep analyses. Surprisingly, very few differences were found between patients with and without RES, despite the large disparity in the Epworth Sleepiness Score. In his editorial comment, RODENSTEIN [2] notes that after such a "breathtaking effort... very little comes out" about what may cause RES. In this scenario, even decidedly unorthodox hypotheses may appear as possible mechanisms for explaining RES, worthy of future investigations. For instance, RODENSTEIN [2] suggested considering the effects of "catastrophising" in perceiving and reporting subjective sleepiness, or the hypothesis that diabetes plays a protective role in RES [2].

We would like to suggest, however, two more orthodox explanations for RES in OSA patients properly treated by CPAP. The first explanation regards the presence of the obesity hypoventilation syndrome (OHS), or of a comorbidity with chronic obstructive pulmonary disease, the so-called overlap syndrome (OS), in the OSA group with RES. In fact, both OHS and OS contribute to daytime sleepiness [3, 4] through hypercapnia. Proper CPAP treatment in OSA patients with OHS may make the sleep architecture, the arousal index and the apnoea/hypopnoea index similar to those of CPAP-treated OSA patients without OHS, but nevertheless, hypercapnia may remain significant [5]. Moreover, CPAP treatment does not reduce the high Epworth Sleepiness Score of OS patients [4]. Since arterial carbon dioxide tension was not measured, OHS or OS cannot be excluded in the obese OSA patients with RES studied by VERNET et al. [1]. Therefore, RES could be in part explained by the ineffectiveness of CPAP to reduce hypercapnia in OHS or OS patients.
Another explanation for RES could be linked to the possible presence of cardiovascular autonomic dysregulations induced by autonomic or "subcortical" arousals. Autonomic arousals involve brainstem neurons controlling both sleep/vigilance and cardiovascular regulation. They are not necessarily associated with detectable electroencephalographic changes, but are responsible for changes in cardiac autonomic regulation. We have found an association between excessive sleepiness during the day and impaired autonomic cardiac modulation at night when comparing two groups of sleepdisordered breathing patients, with and without excessive daytime sleepiness [6]. Both groups had a wide spectrum of disease severity, from simple snoring to OSA, had similar sleep architecture, and similar apnoea/hypopnoea index and arousal index; but patients with excessive daytime somnolence showed lower baroreflex sensitivity and a greater ratio of low- to high-frequency heart rate powers at night, both indexes of increased sympathetic/vagal balance [7]. The results were confirmed after having removed obese patients to exclude OHS [8]. Thus, cardiovascular autonomic alterations during sleep may be associated with excessive daytime sleepiness, probably because of brainstem dysfunctions involving both alertness and cardiovascular control. Standard polysomnographic studies (like those conducted by VERNET et al. [1]) cannot detect these alterations. If such alterations were found, they would support the speculation of VERNET et al. [1] that intermittent hypoxia causes a post-hypoxic injury determining "central hypersomnia". In fact, this is what can be expected if the injury affects specific neuronal groups in the brainstem.

In conclusion, we think that testing for the presence of hypercapnia or of autonomic alterations during sleep could importantly improve understanding of the mechanisms responsible for RES in CPAP-treated OSA patients.

\section{P. Castiglioni* , C. Lombardi ${ }^{\#}$, P. Cortelli ${ }^{\oplus}$ and G. Parati ${ }^{\#,+}$}

*Fondazione Don C. Gnocchi, "Dept of Cardiology, Centre for Sleep Disorders, S. Luca Hospital, IRCCS, Istituto Auxologico 
Italiano, and ${ }^{+}$Dept of Clinical Medicine and Prevention, University of Milano-Bicocca, Milan, and "Centre for Sleep Disorders, Dept of Neurological Sciences, University of Bologna, Bologna, Italy.

Correspondence: P. Castiglioni, Fondazione Don C. Gnocchi, via Capecelatro 66, Milan, Italy, 20148. E-mail: pcastiglioni@ dongnocchi.it

Statement of Interest: None declared.

\section{REFERENCES}

1 Vernet C, Redolfi S, Attali V, et al. Residual sleepiness in obstructive sleep apnoea: phenotype and related symptoms. Eur Respir J 2011; 38: 98-105.

2 Rodenstein D. What is this thing called somnolence? Eur Respir J 2011; 38: 7-8.

3 Hida W, Okabe S, Tatsumi K, et al. Nasal continuous positive airway pressure improves quality of life in obesity hypoventilation syndrome. Sleep Breath 2003; 7: 3-12.

4 Marin JM, Soriano JB, Carrizo SJ, et al. Outcomes in patients with chronic obstructive pulmonary disease and obstructive sleep apnoea: the overlap syndrome. Am J Respir Crit Care Med 2010; 182: 325-331.

5 Banerjee D, Yee BJ, Piper AJ, et al. Obesity hypoventilation syndrome: hypoxemia during continuous positive airway pressure. Chest 2007; 131: 1678-1684.

6 Lombardi C, Parati G, Cortelli P, et al. Daytime sleepiness and neural cardiac modulation in sleep-related breathing disorders. J Sleep Res 2008; 17: 263-270.

7 Bonsignore MR, Parati G, Insalaco G, et al. Baroreflex control of heart rate during sleep in severe obstructive sleep apnoea: effects of acute CPAP. Eur Respir J 2006; 27: 128-135.

8 Castiglioni P, Lombardi C, Di Rienzo M, et al. What are the causes of excessive daytime sleepiness in patients with sleep-disordered breathing? Eur Respir J 2008; 32: 526-527.

\section{DOI: $10.1183 / 09031936.00147111$}

\section{From the authors:}

P. Castiglioni and co-workers suggest that residual excessive sleepiness (RES) in patients with obstructive sleep apnoea (OSA) who are adequately treated with nasal positive airway pressure may be caused by a concomitant, untreated hypercapnia (if OSA overlaps with obesity hypoventilation syndrome or with chronic obstructive pulmonary disease) or by persistent autonomic subcortical arousals. We examined with interest both hypotheses, but neither fits our series [1]. Concomitant chronic obstructive pulmonary disease was a criterion for exclusion (as was any medical disease that could cause sleepiness per se), after reviewing the clinical history and respiratory function tests. As for obesity hypoventilation syndrome, all patients with a body mass index $<30 \mathrm{~kg} \cdot \mathrm{m}^{-2}$ (which represents only half of the sample in the RES group) had arterial blood gases and functional respiratory tests, and none had any hypercapnia (arterial carbon dioxide tension $>45 \mathrm{mmHg}$ ) (table 1). In addition, we noted that the patients with RES were thinner, as a mean, than those without sleepiness.

As for autonomic activity, we performed a spectral analysis of the $\mathrm{R}-\mathrm{R}$ cardiac interval (low and high frequencies) in the three groups of the sample, as suggested. Heart rate spectrograms were obtained by computing $\mathrm{R}-\mathrm{R}$ interval power spectra over a running data window of $110 \mathrm{~s}$. Spectral powers were calculated in the low- (LF; $0.04-0.14 \mathrm{~Hz}$ ) and high-frequency (HF; 0.15$0.40 \mathrm{~Hz}$ ) bands. The ratio between the LF and HF powers of the $\mathrm{R}-\mathrm{R}$ interval (LF/HF) was taken as an indirect measure of cardiac sympathetic/vagal balance [2]. The LF and HF powers and LF/HF power ratio were averaged over the whole recording. None of these measurements were different between treated OSA patients with and without RES or between patients and controls (table 1). In addition, the autonomic activation index (LF/HF ratio) did not significantly correlate with the residual Epworth sleepiness score (Pearson correlation $r=0.25, p=0.17$ ), residual mean sleep latency in the multiple sleep latency test $(\mathrm{r}=-0.16, \mathrm{p}=0.37)$ or fatigue score $(\mathrm{r}=-0.07, \mathrm{p}=0.69)$ in the apnoeic groups. Therefore, the association between high LF/HF ratios (indicating high sympathetic activity during the night) and sleepiness (measured by the multiple sleep latency test) previously found in untreated OSA patients [3] was probably related to untreated respiratory events. When respiratory events are efficaciously treated, as in our sample, the LF/HF ratios are similar to those of controls and do not correlate with any measure of sleepiness or fatigue. This new result provides additional evidence to support the concept that residual sleepiness (and in our study, the feeling of nonrestorative sleep) is not the

TABLE 1 Measurements of arterial carbon dioxide tension $\left(\mathrm{Pa}_{2} \mathrm{CO}_{2}\right)$ and spectral analysis of the R-R cardiac interval in patients with obstructive sleep apnoea (OSA) with and without residual excessive sleepiness (RES) despite adequate positive airway pressure, and healthy controls

\begin{tabular}{|c|c|c|c|c|}
\hline Subjects n & 20 & 20 & 20 & \\
\hline $\mathrm{Pa}, \mathrm{CO}_{2} \mathrm{mmHg}$ (range) & $39.7 \pm 4.9(34-43)$ & $40.3 \pm 2.5(36.6-45)$ & ND & 0.86 \\
\hline $\mathrm{LF} \mathrm{ms}^{2}$ & $369 \pm 291$ & $458 \pm 577$ & $379 \pm 277$ & 0.80 \\
\hline $\mathrm{HF} \mathrm{ms}^{2}$ & $366 \pm 687$ & $504 \pm 774$ & $290 \pm 260$ & 0.62 \\
\hline
\end{tabular}

Data are presented as mean \pm SD, unless otherwise stated. BMI: body mass index; LF: low-frequency power for R-R interval; HF: high-frequency power for R-R interval; ND: not determined. *: $p<0.05$ for OSA patients with versus without RES; ${ }^{\prime} p=0.05$ for OSA patients without RES versus healthy controls. 